\title{
Optical Measurement of Cell Membrane Tension
}

\author{
Gabriel Popescu, ${ }^{1}$ Takahiro Ikeda, ${ }^{2}$ Keisuke Goda,${ }^{3}$ Catherine A. Best-Popescu, ${ }^{4}$ Michael Laposata, ${ }^{4}$ Suliana Manley, ${ }^{5}$ \\ Ramachandra R. Dasari, ${ }^{1}$ Kamran Badizadegan, ${ }^{1,4}$ and Michael S. Feld ${ }^{1}$ \\ ${ }^{1}$ George R. Harrison Spectroscopy Laboratory, Massachusetts Institute of Technology, Cambridge, Massachusetts 02139, USA \\ ${ }^{2}$ Hamamatsu Photonics K.K., 5000 Hirakuchi, Hamamatsu, Shizuoka 434-8601 Japan \\ ${ }^{3}$ Department of Physics, Massachusetts Institute of Technology, Cambridge, Massachusetts 02139, USA \\ ${ }^{4}$ Department of Pathology, Massachusetts General Hospital and Harvard Medical School, Boston, Massachusetts 02114, USA \\ ${ }^{5}$ Department of Chemical Engineering, Massachusetts Institute of Technology, Cambridge, Massachusetts 02139, USA
}

(Received 16 April 2006; published 20 November 2006)

\begin{abstract}
Using a novel noncontact technique based on optical interferometry, we quantify the nanoscale thermal fluctuations of red blood cells (RBCs) and giant unilamellar vesicles (GUVs). The measurements reveal a nonvanishing tension coefficient for RBCs, which increases as cells transition from a discocytic shape to a spherical shape. The tension coefficient measured for GUVs is, however, a factor of 4-24 smaller. By contrast, the bending moduli for cells and vesicles have similar values. This is consistent with the cytoskeleton confinement model, in which the cytoskeleton inhibits membrane fluctuations [Gov et al., Phys. Rev. Lett. 90, 228101, (2003)].
\end{abstract}

PACS numbers: $87.68 .+z, 87.15$.Ya

The red blood cell (RBC) has a composite membrane consisting of a lipid bilayer coupled to a two-dimensional spectrin network, which grants the cell its characteristic properties of both softness and strong shear elasticity [1,2]. Because mature RBCs lack nuclei, they represent a convenient model for studying cell membranes, which have broad applications in both science and technology $[3,4]$. The lipid bilayer is $4-5 \mathrm{~nm}$ thick, and exhibits fluidlike behavior, characterized by a finite bending modulus $\kappa$ and a vanishing shear modulus, $\mu \approx 0$. The resistance to shear, crucial for RBC function, is provided by the spectrin network, which has a mesh size of $\sim 80 \mathrm{~nm}$.

Spontaneous membrane fluctuations, or "flickering," have been modeled theoretically under both static and dynamic conditions [5-9]. These thermally induced membrane motions exhibit $100 \mathrm{~nm}$ scale amplitudes at frequencies of tens of Hz. In past studies, measurements of the membrane mean squared displacement versus spatial wave vector, $\Delta u^{2}(q)$, revealed a $q^{-4}$ dependence predicted by the equipartition theorem, which is indicative of fluidlike behavior $[6,10-13]$. These results conflict with the static deformation measurements provided by micropipette aspiration $[14,15]$, high-frequency electric fields [16,17], and, more recently, optical tweezers [18], which indicate an average value for the shear elasticity of the order of $\mu \sim$ $10^{-6} \mathrm{~J} / \mathrm{m}^{2}$. Gov et al. predicted that the cytoskeleton pinning of the membrane has an overall effect of confining the fluctuations and, thus, gives rise to superficial tension much larger than in the case of free bilayers [5]. This confinement model may offer new insight into the cytoskeleton-bilayer interaction that determines the morphology and physiology of the cell [19].

RBCs can be assumed optically homogeneous. Therefore, measurement of the cell optical path length via interferometric techniques can provide information about the physical topography of the membrane with sub- wavelength accuracy and without contact. However, existing optical methods, including phase contrast microscopy (PCM) [6], reflection interference contrast microscopy (RICM) [10], and fluorescence interference contrast (FLIC) [20], are limited in their ability to measure cell membrane displacements. PCM provides phase shifts quantitatively only for samples that are optically much thinner than the wavelength of light, which is a condition hardly satisfied by any cell type. Similarly, a single RICM measurement cannot provide the absolute cell thickness unless additional measurements or approximations are made [21]. FLIC relies on inferring the position of fluorescent dye molecules attached to the membrane from the absolute fluorescence intensity, which may limit both the sensitivity and acquisition rate of the technique [20].

In this Letter, we present highly sensitive experimental measurements of thermal fluctuations associated with GUVs and RBCs under different morphological conditions. Our experimental method combines Hilbert phase microscopy (HPM) [21,22] developed in our laboratory with an electronic stabilization feedback loop and is referred to as the stabilized Hilbert phase microscopy (sHPM). The experimental geometry is depicted in Fig. 1. The $632.8 \mathrm{~nm}$ radiation from a HeNe laser is collimated and divided into a sample $(S)$ and reference $(R)$ arm to form a modified Mach-Zender interferometer. The sample beam provides the illumination field for an inverted microscope equipped with an immersion $100 \times$ $(\mathrm{NA}=1.2)$ objective that allows for a transverse resolution of $0.26 \mu \mathrm{m}$. The tube lens is positioned such that the image of the sample is formed at the CCD plane via the beam splitter cube. The reference field is collimated and expanded by a telescopic system consisting of another microscope objective and the same tube lens. At the CCD plane, this reference beam can be approximated by a plane wave, which interferes with the image field. The 


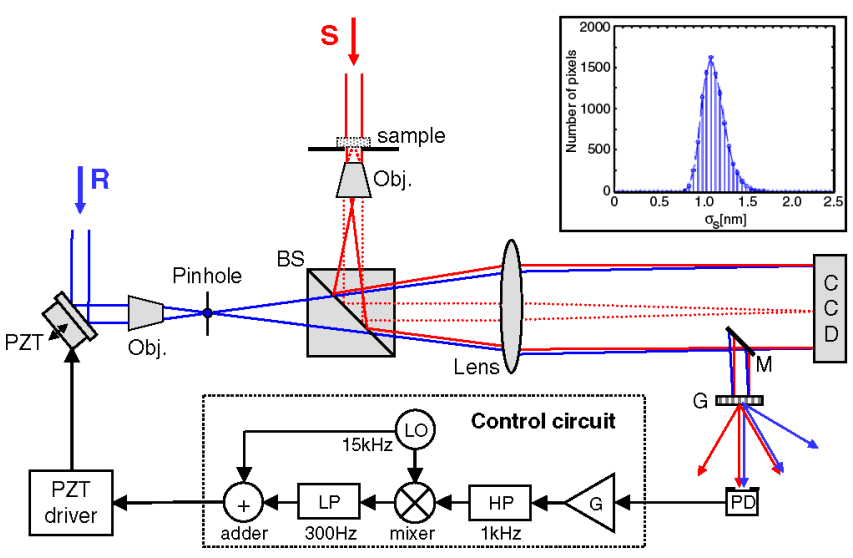

FIG. 1 (color online). Stabilized Hilbert phase microscope. PZT, piezoelectric transducer; Obj, microscope objective; BS, beam splitter cube; $M$, mirror; $\mathrm{PD}$, photodetector; $G$, amplifier; $\mathrm{HP}$, high-pass filter; LP, low-pass filter.

reference field is tilted with respect to the sample field such that uniform fringes are created at an angle of $45^{\circ}$ with respect to the $x$ and $y$ axes. The CCD used (C7770, Hamamatsu Photonics) has an acquisition rate of 291 frames/s with $640 \times 480$ pixels per image the fringes are sampled by 4-6 CCD pixels per period. Using highpass spatial filtering and Hilbert transformation, the spatially varying phase $\phi(x, y)$ associated with the microscope image is retrieved in each point from a single-exposure image [23]. In order to suppress the inherent phase noise, we used a stabilization feedback system that locks the interferometer on an interference fringe, as follows. A small mirror $M$ deflects a portion of the interfering beams before they reach the CCD. At a plane conjugate to the image (CCD) plane, we place an amplitude grating, which has the same period as the interferogram. Because of this spatial matching, each diffraction order $n$ produced by the sample beam overlaps with the order $n-1$ of the reference beam. The two interfering beams propagating on axis are spatially isolated by an aperture and detected by the photodiode PD. The feedback loop operates on a principle similar to that described in Ref. [24]. The PD signal is mixed with the local oscillator (LO) that modulates the reference arm length, and then is low-pass filtered, yielding the error signal. The frequency of the LO is chosen to be $\Omega /(2 \pi)=15 \mathrm{kHz}$ such that the displacements of the sample can be measured up to about $1 \mathrm{kHz}$. The control signal that corrects the reference arm length with respect to the sample arm against the fringe fluctuations is combined with the LO modulation signal and fed to the PZT. The stability of the instrument against the residual noise was assessed by acquiring successive phase images of the field of view with no sample. The optical path-length standard deviation $\sigma_{s}$ calculated from 128 phase images at $10 \mathrm{~ms} /$ frame was calculated for each pixel. The inset of Fig. 1 shows the histogram of the standard deviation obtained from an area of $100 \times 100$ pixels. The average standard deviation had a value of $1.2 \mathrm{~nm}$, which demon- strates the efficacy of the active stabilization. This combination of HPM and active stabilization allows for accurate quantitative measurements of cell membrane fluctuations and represents a significant improvement with respect to our previous reports [22,23].

In order to investigate the physical difference between RBC membrane and free bilayer fluctuations, we measured the fluctuations of GUVs. The membrane composition we used was $100 \%$ SOPC (1-Stearoyl-2-Oleoyl-sn-Glycero-3Phosphocholine). The GUVs were electroformed for 2 hours under a $1 \mathrm{~V}, 10 \mathrm{~Hz}$ ac field in a $300 \mathrm{mM}$ glucose solution [25] and diluted in a sucrose solution for increased optical contrast, and to slightly deflate the vesicles. The refractive index contrast with respect to the surrounding fluid, $\Delta n=0.011$, was obtained from the quantitative phase image by assuming a spherical profile for the vesicles. Sets of 1000 time-resolved phase images of individual GUVs were recorded at $10.3 \mathrm{~ms} /$ frame. The vesicles under investigation ranged in diameter from 8-12 $\mu \mathrm{m}$ and their thickness profile was obtained from phase images as $u(x, y ; t)=(\lambda / 2 \pi \Delta n) \phi(x, y ; t)$. Each 1000 frame data set was then separated into groups of 128 frames, analyzed separately to obtain mean squared displacements, and averaged to provide statistically significant information for each vesicle. Using Fourier transformations both in time and space, we obtained the mean squared displacement as a function of spatial wave vector and temporal frequency, $\Delta u^{2}(\mathbf{q}, \omega)$. Figure 2 summarizes the static (spatial) behavior of the thermal fluctuations averaged over 5 vesicles, $\Delta u^{2}(q)=\int \Delta u^{2}(q, \omega) d \omega$, with $q=|\mathbf{q}|$. The data can be fitted very well over the spatial wave vector interval $1<q<13 \mathrm{rad} / \mu \mathrm{m}$ by the equation [6]

$$
\Delta u^{2}(q)=\frac{k_{B} T}{\kappa q^{4}+\sigma q^{2}},
$$

where $k_{B}$ is the Boltzmann constant and $T=298 \mathrm{~K}$ is the absolute temperature for our experiments. The measured values at low- $q$ deviate from this form; however, this is expected for wavelengths corresponding to the size of the vesicles. The only two parameters used for the fit are the tension coefficient, $\sigma$, and the bending modulus, $\kappa$. The value for the bending modulus obtained from the fit is $\kappa=$ $(0.7 \pm 0.12) \times 10^{-20} \mathrm{~J}$ and the tension coefficient was $\sigma=(3.5 \pm 0.6) \times 10^{-7} \mathrm{~J} / \mathrm{m}^{2}$. These values are in good agreement with those obtained using pipette aspiration [26].

Blood samples were collected and centrifuged for $10 \mathrm{mi}-$ nutes at $2000 \mathrm{~g}$ and $5^{\circ} \mathrm{C}$ to separate RBCs from plasma. The cells were washed 3 times with saline and suspended

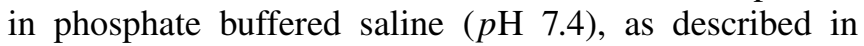
Ref. [27]. The RBCs were then placed between cover slips and imaged without additional preparation. Using a refractive index contrast $\Delta n=6 \%$ between the hemoglobin solution contained in the cell and the surrounding fluid [28], the RBC thickness profile was directly obtained from 


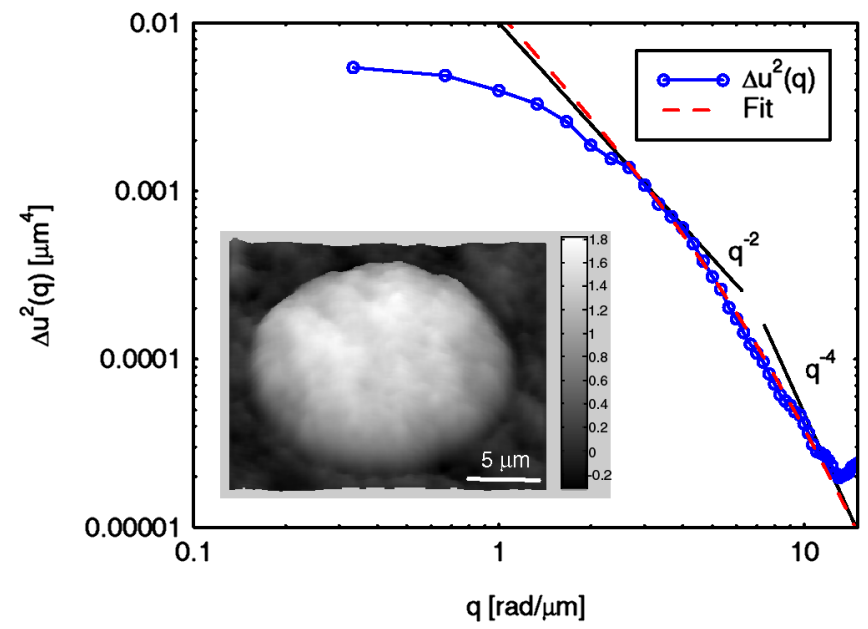

FIG. 2 (color online). Mean squared displacements of giant unilamellar vesicles fitted with Eq. (1), as indicated. The solid lines indicate the asymptotic behavior. The inset shows the phase image of a vesicle, with the gray scale in radians.

the phase image. The membrane displacements were statistically analyzed both spatially and temporally.

Our samples were primarily composed of RBCs with a typical discocytic shape, but also contained cells with abnormal morphology which formed spontaneously in the suspension, such as echinocytes, with a spiculated shape, and spherocytes, approaching a spherical shape. By taking into account the free energy contributions of both the bilayer and cytoskeleton, these morphological changes have been successfully modeled [19]. Figures 3(a)-3(c) show typical sHPM images of cells in these three groups. For comparison, we also analyzed the motions of RBCs fixed with $40 \mu \mathrm{M}$ gluteraldehyde using a standard procedure [29]. The resultant mean squared displacements, $\Delta u^{2}(q)$, for each group of $4-5$ cells, are summarized in Fig. 3(d). The fixed cells show significantly diminished fluctuations, as expected. The curves associated with the three untreated RBC groups exhibit a power law behavior with an exponent $\alpha=2$. As in the case of vesicles, this dependence is an indication of tension; however, the RBC tension is determined by the confinement of the bilayer by the cytoskeleton $[5,30]$. Based on this model, we fitted the data to extract the tension coefficient for each individual cell. The average values obtained for the discocytes, echinocytes, and spherocytes are, respectively, $\sigma=$ $(1.5 \pm 0.2) \times 10^{-6} \mathrm{~J} / \mathrm{m}^{2}, \quad \sigma=(4.05 \pm 1.1) \times 10^{-6} \mathrm{~J} / \mathrm{m}^{2}$, and $\sigma=(8.25 \pm 1.6) \times 10^{-6} \mathrm{~J} / \mathrm{m}^{2}$. Thus, our data indicate the existence of positive tension in the membrane, which agree very well with the simulation by Discher et al. [31]. The tension coefficient of RBCs is 4-24 times larger than that of vesicles, which suggests that the contribution of the cytoskeleton might be responsible for this enhancement. Further, it is known that the cytoskeleton plays a role in the transitions from a normal red blood cell shape to abnormal morphology, such as echinocyte and spherocyte [19]. Therefore, the consistent increase in tension for the discocyte-echinocyte-spherocyte transition can be explained by changes in the cytoskeleton, which pins the bilayer. These findings support the hypothesis that the fluctuations are laterally confined by a characteristic length, $\xi_{0}=2 \pi \sqrt{\kappa / \sigma}$, which is much smaller than the cell size [5]. We propose that this characteristic length scale is directly measurable as a transverse coherence length of the membrane motions. In this picture, by pinning the bilayer, the cytoskeleton defines a certain coherence area of the fluctuations, which is inversely proportional to tension. In order to calculate the coherence area of cell membranes, we applied definitions from the coherence theory of optical fields in the space-frequency domain [32]. Thus, the cross-spectral density, $\Delta u^{2}(\rho, \omega)$, is a measure of the spatial correlations between two points separated by a distance $\rho$ at a particular frequency. The power spectrum of the fluctuations can be obtained as $P(\omega)=$ $\Delta u^{2}(0, \omega)$. Figure 4(a) shows the power spectra associated with the three groups of RBCs. All curves exhibit power law behavior $\omega^{-\alpha}$ with slightly different exponent values, $1.15<\alpha<1.54$, which is compatible with $\alpha=1.33$ proposed in the literature $[6,30]$. Using the measured crossspectral density, we calculated the spectral transverse coherence length as

$$
\xi(\omega)=\sqrt{\frac{\int \rho^{2} \Delta u^{2}(\rho, \omega) \rho d \rho}{\int \Delta u^{2}(\rho, \omega) \rho d \rho}} .
$$

The transverse coherence length $\xi_{0}$ was obtained by averaging $\xi(\omega)$ over frequency. This information was used to extract the bending modulus of the RBCs, given by $\kappa=$ $\sigma\left[\xi_{0} /(2 \pi)\right]^{2}$. Figure 4 (b) shows the values measured for both the tension coefficient and bending modulus versus the root mean squared (rms) displacement, $\Delta u_{\mathrm{rms}}=$ $\sqrt{2 \pi \int \Delta u^{2}(q) q d q}$. Remarkably, the bending moduli for the different red blood cell shapes have similar values, $\kappa=$ $(0.75 \pm 0.15) \times 10^{-20}$. This result supports our hypothesis
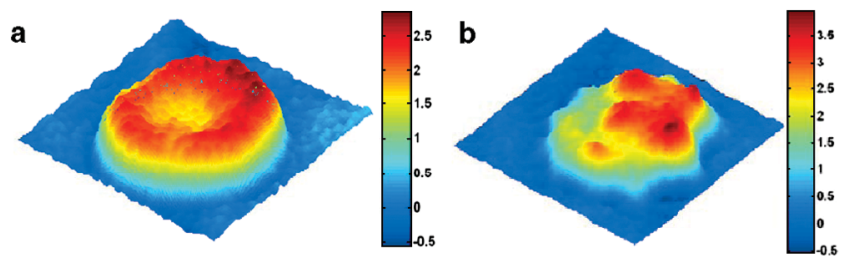

C
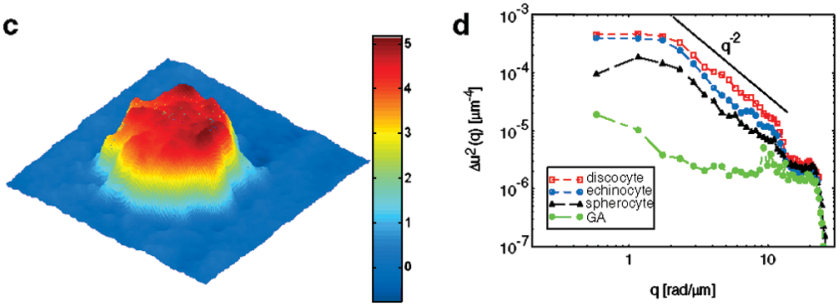

FIG. 3 (color). (a)-(c) sHPM images of a discocyte (a), echinocyte (b), and spherocyte (c). The color bar shows thickness in microns. (d) Mean squared displacements for the three RBC groups and for the gluteraldehyde (GA) fixed cells. 

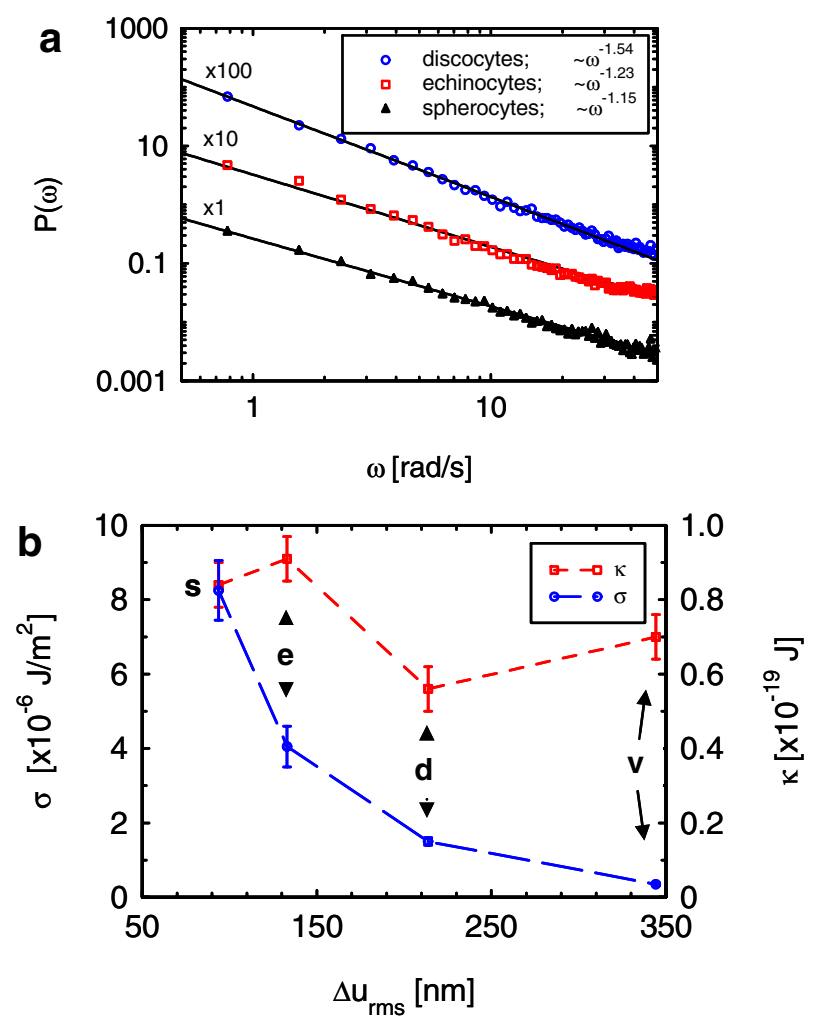

FIG. 4 (color online). (a) Power spectrum associated with the three RBC groups, as indicated. For clarity, the curves are scaled by different factors, as shown. The solid lines represent fits with the power law functions indicated in the legend. (b) Tension factor and bending modulus vs the rms fluctuations for RBCs of different shapes and vesicles: $s$ : spherocyte, $e$ : echinocyte, $d$ : discocyte, $v$ : vesicle.

that the difference in the fluctuations can be explained by renormalizing only one parameter, the characteristic length, which defines the coherence area of the motions. In addition, the $\kappa$ values obtained for all the RBC groups are in very good agreement with those for vesicles, which suggests that the slight differences in composition between the two structures do not affect significantly their bending properties.

In summary, our measurements demonstrate the existence of tension in RBCs of various shapes and GUVs. The tension coefficient measured in red blood cells has significantly larger values than in vesicles and increases as the cell transitions from a discocyte to a spherocyte. These findings demonstrate the effect of the cytoskeleton, which can be accounted for by a proper renormalization of the coherence area of the fluctuations. Compared to other optical techniques used for studying membrane fluctuations, the sHPM technique used here is quantitative in terms of membrane topography and displacements, highly sensitive to the nanoscale membrane motions, and provides high transverse resolution.
[1] D. Boal, Mechanics of the Cell (Cambridge University Press, Cambridge, England, 2002).

[2] R.M. Hochmuth and R.E. Waugh, Annual Review of Physiology 49, 209 (1987).

[3] R. Lipowsky, Nature (London) 349, 475 (1991).

[4] E. Sackmann, Science 271, 43 (1996).

[5] N. Gov, A. G. Zilman, and S. Safran, Phys. Rev. Lett. 90, 228101 (2003).

[6] F. Brochard and J. F. Lennon, J. Phys. (France) 36, 1035 (1975).

[7] N. Gov, Phys. Rev. Lett. 93, 268104 (2004).

[8] N. S. Gov and S. A. Safran, Biophys. J. 88, 1859 (2005).

[9] R. Lipowski and M. Girardet, Phys. Rev. Lett. 65, 2893 (1990).

[10] A. Zilker, H. Engelhardt, and E. Sackmann, J. Phys. (France) 48, 2139 (1987).

[11] K. Zeman, H. Engelhardt, and E. Sackman, Eur. Biophys. J. 18, 203 (1990).

[12] A. Zilker, M. Ziegler, and E. Sackmann, Phys. Rev. A 46, 7998 (1992).

[13] H. Strey, M. Peterson, and E. Sackmann, Biophys. J. 69, 478 (1995).

[14] D.E. Discher, N. Mohandas, and E. A. Evans, Science 266, 1032 (1994).

[15] R. M. Hochmuth, P. R. Worthy, and E. A. Evans, Biophys. J. 26, 101 (1979).

[16] H. Engelhardt and E. Sackmann, Biophys. J. 54, 495 (1988).

[17] H. Engelhardt, H. Gaub, and E. Sackmann, Nature (London) 307, 378 (1984).

[18] S. Suresh, J. Spatz, and J.P. Mills et al., Acta Biomaterialia 1, 15 (2005).

[19] H. W. G. Lim, M. Wortis, and R. Mukhopadhyay, Proc. Natl. Acad. Sci. U.S.A. 99, 16766 (2002).

[20] Y. Kaizuka and J. T. Groves, Phys. Rev. Lett. 96, 118101 (2006).

[21] A. Zidovska and E. Sackmann, Phys. Rev. Lett. 96, 048103 (2006).

[22] G. Popescu, T. Ikeda, and C. A. Best et al., J. Biomed. Opt. Lett. 10, 060503 (2005).

[23] T. Ikeda, G. Popescu, and R. R. Dasari et al., Opt. Lett. 30, 1165 (2005).

[24] R. W. P. Drever, J. L. Hall, and F. V. Kowalski et al., Appl. Phys. B 31, 97 (1983).

[25] M. I. Angelova, S. Soleau, and P. Meleard et al., Prog. Colloid Polym. Sci. 89, 122 (1992).

[26] E. Evans and W. Rawicz, Phys. Rev. Lett. 64, 2094 (1990).

[27] C. A. Best, J.E. Cluette-Brown, and M. Teruya et al., J. Lipid Res. 44, 612 (2003).

[28] M. Hammer, D. Schweitzer, and B. Michel et al., Appl. Opt. 37, 7410 (1998).

[29] M. Abkarian, M. Faivre, and H. A. Stone, Proc. Natl. Acad. Sci. U.S.A. 103, 538 (2006).

[30] N. Gov, A. Zilman, and S. Safran, Biophys. J. 84, 486A (2003).

[31] D. E. Discher, D. H. Boal, and S. K. Boey, Biophys. J. 75, 1584 (1998).

[32] L. Mandel and E. Wolf, Optical Coherence and Quantum Optics (Cambridge University Press, Cambridge, England, 1995). 